\title{
Profiling the Salivary microbiome of the Qatari population
}

\author{
Selvasankar Murugesan ${ }^{1}$, Sara Fahad Al Ahmad ${ }^{2}$, Parul Singh ${ }^{1}$, Marwa Saadaoui ${ }^{1}$, Manoj Kumar ${ }^{1}$ \\ and Souhaila Al Khodor ${ }^{\text {** }}$
}

\begin{abstract}
Background: The role of the human microbiome in human health and disease has been studied in various body sites. However, compared to the gut microbiome, where most of the research focus is, the salivary microbiome still bears a vast amount of information that needs to be revealed. This study aims to characterize the salivary microbiome composition in the Qatari population, and to explore specific microbial signatures that can be associated with various lifestyles and different oral conditions.
\end{abstract}

Materials and methods: We characterized the salivary microbiome of 997 Qatari adults using high-throughput sequencing of the $\mathrm{V} 1-\mathrm{V} 3$ region of the $16 \mathrm{~S}$ rRNA gene.

Results: In this study, we have characterized the salivary microbiome of 997 Qatari participants. Our data show that Bacteroidetes, Firmicutes, Actinobacteria and Proteobacteria are the common phyla isolated from the saliva samples, with Bacteroidetes being the most predominant phylum. Bacteroidetes was also more predominant in males versus females in the study cohort, although differences in the microbial diversity were not statistically significant. We also show that, a lower diversity of the salivary microbiome is observed in the elderly participants, with Prevotella and Treponema being the most significant genera. In participants with oral conditions such as mouth ulcers, bleeding or painful gum, our data show that Prevotella and Capnocytophaga are the most dominant genera as compared to the controls. Similar patterns were observed in participants with various smoking habits as compared to the non-smoking participants. Our data show that Streptococcus and Neisseria are more dominant among denture users, as compared to the non-denture users. Our data also show that, abnormal oral conditions are associated with a reduced microbial diversity and microbial richness. Moreover, in this study we show that frequent coffee drinkers have higher microbial diversity compared to the non-drinkers, indicating that coffee may cause changes to the salivary microbiome. Furthermore, tea drinkers show higher microbial richness as compared to the non-tea drinkers.

Conclusion: This is the first study to assess the salivary microbiome in an Arab population, and one of the largest population-based studies aiming to the characterize the salivary microbiome composition and its association with age, oral health, denture use, smoking and coffee-tea consumption.

Keywords: 165 rRNA gene sequencing, Saliva, Dysbiosis, Qatari, Oral health, Qatar Biobank

*Correspondence: salkhodor@sidra.org

1 Research Department, Sidra Medicine, Doha, Qatar

Full list of author information is available at the end of the article

\section{Background}

The human microbiota is the collection of a wide array of microorganisms such as bacteria, archaea, fungi and viruses that inhabit various body sites including skin, saliva and the gut [1]. The microbiome, defined as the collection of microbiota and their genes, plays an important role in human health and disease [2]. The development in 
the field of sequencing and bioinformatic tools in the last decade, has brought an unprecedented attraction to the microbiome field [3]. The microbiome composition varies from one person to another, as well as across different body sites [4].

Saliva is a biofluid secreted by the major and minor salivary glands [5]. It contains several components such as electrolytes, proteins, immunoglobulins, enzymes and microbes [6]. The main role of the saliva is to protect the mucus from pathogens, to maintain tooth integrity in addition to its role in taste and digestion [7]. Being highly available, saliva is considered as an easy to collect sample that does not require hospitalization or special preparation $[8,9]$.

Located at the opening of the gastrointestinal tract, the oral cavity provides a convenient, accessible site for collecting and analyzing microbial samples in the saliva [10]. It is also worth noting that, the salivary microbiome mirrors the gut microbiome in terms of complexity and diversity [10]. The salivary microbiome exhibits longterm stability and does not fluctuate according to the circadian rhythm, indicating that the time of the saliva sampling is not critical [11-16]. Collection of saliva can be achieved by several methods, including spitting, swabbing and draining [17], and the method of collection exerts a minimal effect on the extraction of microbial DNA [18]. A healthy adult human mouth hosts a complex and resilient ecosystem of hundreds of different microbial species [19]. These microbes reside in different sites of the oral cavity, which is mainly composed of a soft mucosa that is constantly shedding, and a hard surface which comprises the teeth [20]. The saliva is a representing constituent of both locations but more profoundly of the soft mucosa [20]. According to many studies, the salivary microbiome consists of more than 700 species mainly belonging to the Bacteroidetes, Firmicutes, Proteobacteria, Actinobacteria and Fusobacteria phyla [21-24]. Streptococcus, Prevotella, Neisseria, Haemophilus, Porphyromonas and Rothia are the common genera members of salivary microbiome observed in healthy adults [25-27]. The salivary microbiome composition is influenced by several pre and post-natal factors including host genetics, the mode of delivery at birth; the method of infant feeding; teeth eruption, the use of medications, especially antibiotics; smoking, intraoral $\mathrm{pH}$, oral hygiene and diet among others [28]. The salivary microbiome plays a major role in regulating the immune-inflammatory balance in the host [29]. In a large American cohort study, $\mathrm{Wu}$ et al. compared the salivary microbiome composition in current smokers and non-smokers [30]. They observed that the salivary microbiome of smokers reflected a decrease in the abundance of the phylum Proteobacteria, and in Capnocytophaga, Peptostreptococcus and Leptotrichia genera; while the genera Atopobium and Streptococcus were found to be elevated in smokers compared to non-smokers [30]. Another study examined the oral microbiome of smokers and non-smokers in addition to the levels of cytokines in saliva samples, where they found that smoking altered the cytokine levels and the salivary microbiome composition [31].

Dysbiosis of the oral microbiome has been implicated in various oral disorders such as periodontitis, tooth decay or loss of teeth, where it promotes pathogenic bacterial growth and enables the dissemination of the oral bacteria systemically [32]. Several studies were conducted in order to assess the microbiome composition and its role in dental and periodontal health, and showed that, healthy individuals have a greater microbial diversity and a greater abundance of Neisseria, Haemophilus, and Fusobacterium. This is in contrast to individuals who suffer from dental caries, where Streptococcus was the most abundant genus detected [33, 34]. In a comparative study of healthy Finnish adults with and without caries, Corynebacterium, Fusobacterium, Capnocytophaga, Porphyromonas, Prevotella, and Leptotrichia were significantly more abundant in healthy volunteers as compared to those with dental caries [35].

Porphyromonas gingivalis, Tannerella forsythia, Treponema denticola, Prevotella intermedia and Aggregatibacter actinomycetemcomitans were shown to be higher in Moroccan patients with periodontitis [36] and P. gingivalis, P. intermedia, T. forsythia and Fretibacterium were higher in Japanese patients with periodontitis $[37,38]$. Moreover, oral microbial dysbiosis is usually observed in patients with systemic diseases such as obesity, diabetes, cancer, rheumatoid arthritis, Parkinson's disease, type 2 diabetes (T2D) and cardiovascular diseases among others [39-45].

Based on its potential role in health and disease, the salivary microbiome harbors a great potential for being used as a health monitor or disease diagnostic tool. However, the degree of variation at the population level has been assessed in very few studies [12, 46, 47], none of them reflecting the Arab population. The aim of this study is to characterize the salivary microbiome of the Qatari population and to assess the role of gender, age, oral health, smoking and some dietary habits in the salivary microbiome composition.

\section{Results}

\section{Demographic and clinical parameters}

We determined the bacterial compositions in the saliva samples of 997 Qatari adults aged $\geq 18$ years using $16 \mathrm{~S}$ rRNA gene sequencing. The subjects' demographic and clinical characteristics including oral hygiene practices are summarized in Table 1. 
Table 1 Demographic status of studied Qatari population

\begin{tabular}{|c|c|c|c|}
\hline Category & Male & Female & Total \\
\hline Gender & 442 & 555 & 997 \\
\hline $\mathrm{Age}^{\mathrm{a}}$ & $38.3 \pm 11.9$ & $39.3 \pm 12.1$ & $38.2 \pm 12.0$ \\
\hline \multicolumn{4}{|l|}{ Age group } \\
\hline Adults $(18 \geq$ age $\leq 65)$ & 434 (98.2\%) & $545(98.2 \%)$ & 979 (98.2\%) \\
\hline Elderly (age > 65) & $8(1.8 \%)$ & $10(1.8 \%)$ & $18(1.8 \%)$ \\
\hline \multicolumn{4}{|l|}{ Oral health status } \\
\hline \multicolumn{4}{|l|}{ Bleeding gum } \\
\hline No & $398(90.0 \%)$ & $515(92.8 \%)$ & 913 (91.6\%) \\
\hline Yes & $44(10 \%)$ & 40 (7.20\%) & $84(8.4 \%)$ \\
\hline \multicolumn{4}{|l|}{ Mouth ulcer } \\
\hline No & 419 (94.8\%) & $541(97.5 \%)$ & $960(96.3 \%)$ \\
\hline Yes & $23(5.2 \%)$ & $14(2.5 \%)$ & $37(3.7 \%)$ \\
\hline \multicolumn{4}{|l|}{ Painful gum } \\
\hline No & $400(90.5 \%)$ & $516(93.0 \%)$ & 916 (91.9\%) \\
\hline Yes & $42(9.5 \%)$ & $39(7.0 \%)$ & $81(8.1 \%)$ \\
\hline \multicolumn{4}{|l|}{ Loose teeth } \\
\hline No & $422(95.5 \%)$ & $537(96.8 \%)$ & 959 (96.2\%) \\
\hline Yes & $20(4.5 \%)$ & $18(3.2 \%)$ & $38(3.8 \%)$ \\
\hline \multicolumn{4}{|l|}{ Denture } \\
\hline No & $387(87.6 \%)$ & $474(85.4 \%)$ & 861 (86.4\%) \\
\hline Yes & $55(12.4 \%)$ & $81(14.6 \%)$ & $136(13.6 \%)$ \\
\hline \multicolumn{4}{|l|}{ Smoking status } \\
\hline Non-smokers & $210(47.5 \%)$ & $523(94.2 \%)$ & $733(73.5 \%)$ \\
\hline Smokers & $232(52.5 \%)$ & $32(5.8 \%)$ & $264(26.5 \%)$ \\
\hline \multicolumn{4}{|l|}{ Coffee drinking } \\
\hline Coffee & $418(94.6 \%)$ & $469(84.5 \%)$ & 887 (89.0\%) \\
\hline No coffee & $24(5.4 \%)$ & $86(15.5 \%)$ & $110(11.0 \%)$ \\
\hline \multicolumn{4}{|l|}{ Tea drinking } \\
\hline Tea & $363(82.1 \%)$ & 405 (73.0\%) & 768 (77.0\%) \\
\hline No tea & 79 (17.9\%) & $150(27.0 \%)$ & $229(23.0 \%)$ \\
\hline
\end{tabular}

a Age: (years, mean \pm standard deviation)

The salivary microbiome of the Qatari population

From the analysis of $16 \mathrm{~S}$ rRNA gene data, 10 different bacterial phyla and 112 genera were identified in the saliva samples collected form the Qatari participants included in this study. Bacteroidetes (65.9\%), Firmicutes (15.8\%), Proteobacteria (2.7\%), Fusobacteria (0.7\%) and Saccharibacteria $(0.3 \%)$ were the top five phyla observed covering about $85 \%$ of the salivary microbiome composition (Additional file 1: Figure S1A). At the genus levels, our data show that a total of 13 genera (Prevotella, Porphyromonas, Streptococcus, Veillonella, Capnocytophaga, Haemophilus, Gemella, Alloprevotella, Granulicatella, Camphylobacter, Leptotrichia, Megasphaera and Neisseria) were commonly represented in all participants covering $85 \%$ of the salivary microbiome profiles (Additional file 1: Figure S1B). We then compared the salivary microbiome composition of the Qatari population to the salivary microbial profiles in other populations. From NCBI/SRA bioprojects, we retrieved the available salivary microbiome sequences from populations such as Bangladesh, Brazil, Japan, South Korea Germany, UK, and USA. Comparison of the salivary microbial profiles of the above-mentioned populations and the salivary microbiome composition in the samples collected from Qataris, revealed differences in the composition at both the phylum (Additional file 2: Figure S2A, B and data not shown) and genus levels in addition to microbial diversity (Additional file 2: Figure S2C), indicating a population-based variability in the salivary microbial profiles $[12,46,47]$. Bacteroidetes was the predominant bacteria among Qatari and German populations with $72 \%$ and $33 \%$ of the total phyla composition respectively. On the other hand, UK (78.32\%), Brazil (50.25\%), USA (40.62\%), Bangladesh (32.11\%), Japan (42.48\%) and South Korean (39.91\%) populations showed higher abundance of Firmicutes (Additional file 2: Figure S2B). Beta diversity measures using Bray-Curtis distances showed a significant clustering between different populations and this result was confirmed using Anosim test with a $P$ value of 0.001 (Additional file 2: Figure S2C).

\section{Gender and the salivary microbiome}

We assessed whether specific differences in the bacterial taxa were observed between males and females. The relative abundance of the salivary microbiome at the phylum level showed that Bacteroidetes, Firmicutes, Proteobacteria and Fusobacterium were the most common phyla observed in the saliva samples (Fig. 1a). A significant increase of Bacteroidetes was observed in males (67.3\%) as compared to females (64.8\%) (Fig. 1a, c-upper panel). At the genus level, Prevotella, Porphyromonas, Streptococcus and Veillonella were the top abundant members of the salivary microbiome observed in both males and females. Bergeyella, Tannerella genera were significantly higher in males while Treponema, Mycoplasma and Corynebacterium genera were significant higher in females (Fig. 1b, c-lower panel). The indices Chao1, observed OTUs, Shannon index, and Simpson were used to examine alpha diversity (Fig. 1d). Bacterial community richness (Chao1) and diversity (Shannon and Simpson) of the microbiome showed no significant differences between males and females (Fig. 1d). On the other hand, the observed species richness index was significantly higher $(P=0.042)$ among Qatari males than females (Fig. 1d). Beta diversity measures using Bray-Curtis distances did not show any group specific clustering, this result was also confirmed using Anosim test (Fig. 1e). 

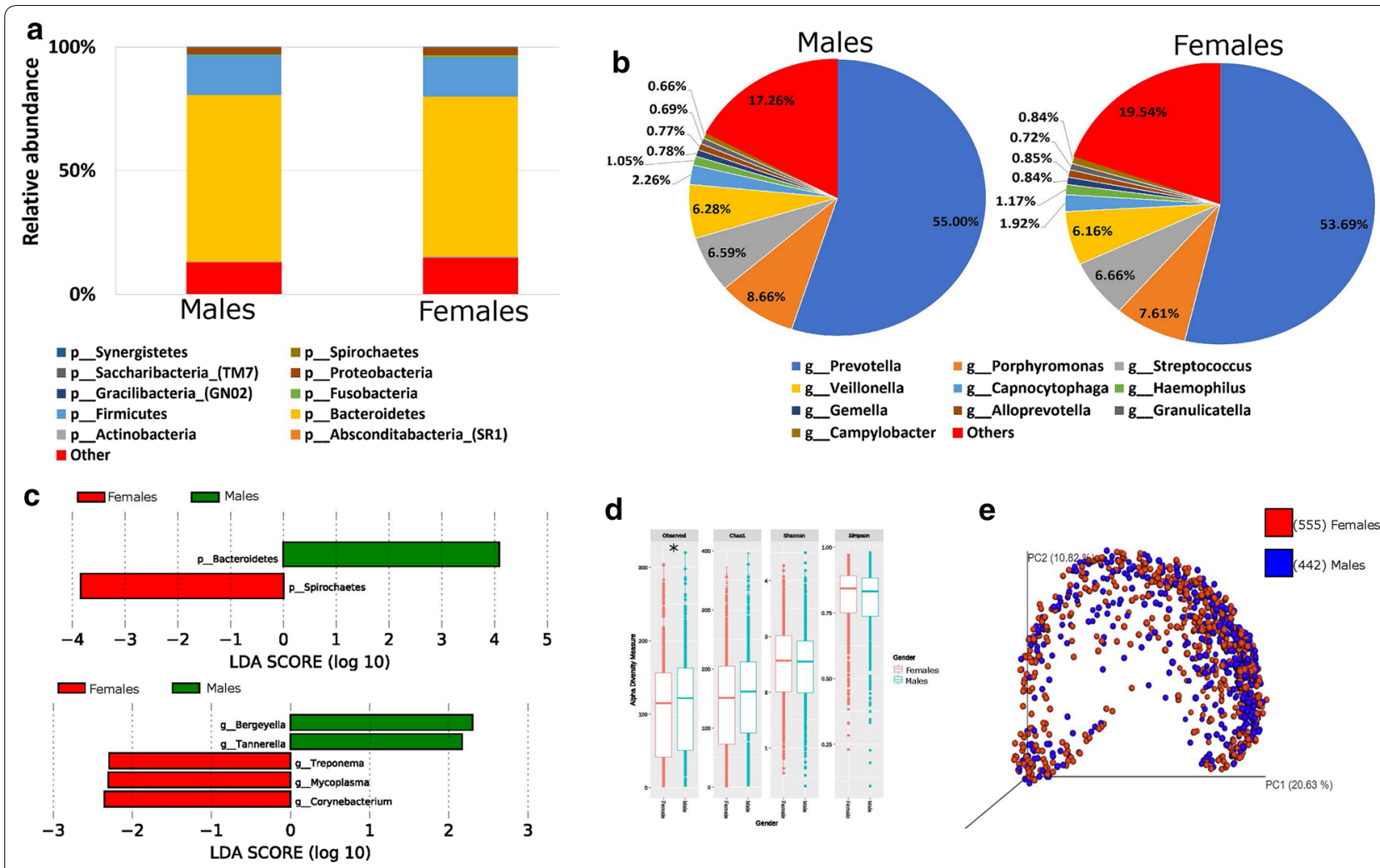

d

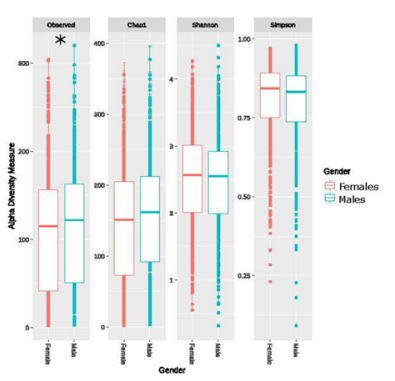

e

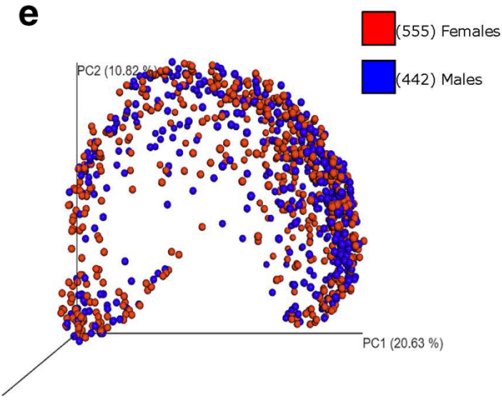

Fig. 1 Gender and the salivary microbiome. Y-axis shows \% of relative abundance; X-axis indicates the microbial abundance in males and females; each taxonomic category is shown by a different color: $\mathbf{a}$ at the phylum level; $\mathbf{b}$ at the genus level; $\mathbf{c}$ graphs of linear discriminant analysis (LDA) scores for differentially abundant bacterial phyla and genera; among the two groups. LDA scores indicate overrepresented bacteria in males (green) and females (red). Features with LDA scores $\geq 2$ are presented. $\mathbf{d}$ Alpha diversity measures for the two groups. Alpha diversity was measured by the number of OTUs observed or by the Chao1, Shannon and Simpson diversity measures; e Principle Coordinates Analysis (PCoA) based on Bray-Curtis dissimilarities of salivary microbiome. Axes are scaled to the amount of variation explained; ${ }^{*} \mathrm{P}<0.05$

\section{The salivary microbiome composition and aging}

Analysis of the relative abundance of the salivary microbiome in older participants (defined as people older than 65 years of age) revealed that Bacteroidetes and Spirochaetes were significantly higher in the elderly participants as compared to their adults' counterparts (less than 65 years old) (Fig. 2a, c-upper panel). On the other hand, Actinobacteria, Firmicutes, Fusobacteria, Proteobacteria and Saccharibacteria were significantly higher in the adults (Fig. 2a, c-upper panel) compared to the older participants at the phylum level. Moreover, analysis at the genus level showed that the abundance of Prevotella and Treponema genera were significantly higher in the elderly participants, whereas, Veillonella, Streptococcus, Mogibacterium, Megasphaera, Rothia and Camphylobacter genera among others were significantly elevated in the adults participants (Fig. 2b, c-lower panel). Alpha-diversity measures indicated that the salivary microbiome in the elderly participants has a lower bacterial richness and diversity as compared to the adults ( $P$ value $<0.001$ ) (Fig. 2d). In Bray-Curtis based beta diversity, although the clustering of the adults' salivary microbiome was not conclusive; the anosim analysis of distance matrices revealed that there is a significant difference among the adults and elderly participants with a $P$ value of 0.007 (Fig. 2e).

\section{The salivary microbiome composition and oral health}

The study participants were stratified according to their answers recorded in the oral health section of the QBB baseline questionnaire. Those questions included whether the participant suffers from a bleeding gum, a mouth ulcer, painful gum, loose teeth, and where they have a denture or not (Table 1). Participants who answered No to all of the oral health-related questions were used as controls.

\section{Bleeding gum and the salivary microbiome composition}

A total of 84 participants reported suffering from a bleeding gum (Table 1). The salivary microbiome composition in those participants was compared to age and gender matching controls. Our analysis revealed that 

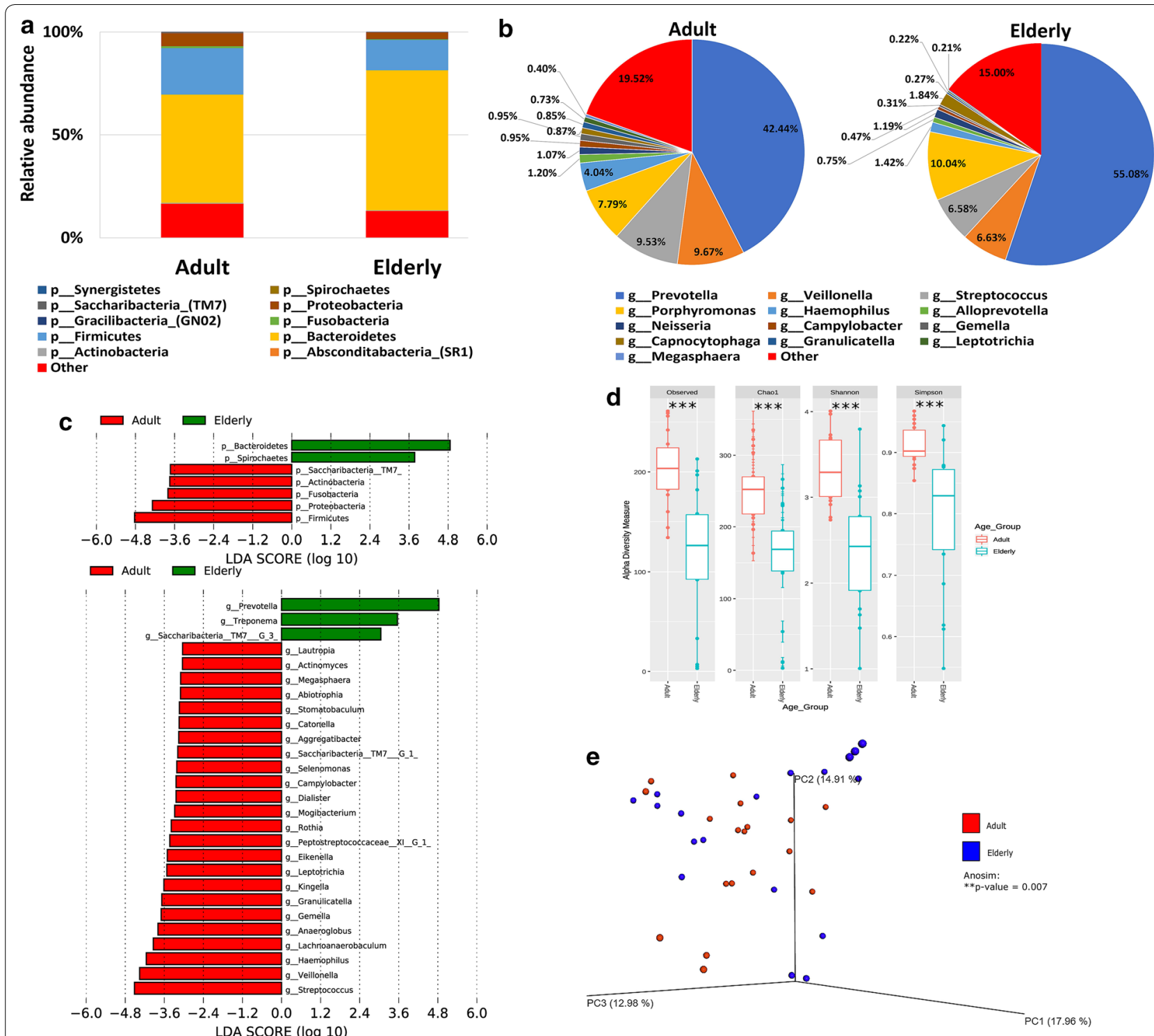

Fig. 2 The salivary microbiome composition and aging. Y-axis shows \% of relative abundance; X-axis indicates the microbial abundance in males and females; each taxonomic category is shown by a different color: $\mathbf{a}$ at the phylum level; $\mathbf{b}$ at the genus level; c graphs of linear discriminant analysis (LDA) scores for differentially abundant bacterial phyla and genera; among the two groups. LDA scores indicate overrepresented bacteria in Elderly (green) and Adults (red). Features with LDA scores $\geq 2$ are presented. $\mathbf{d}$ Alpha diversity measures for the two groups. Alpha diversity was measured by the number of OTUs observed or by the Chao1, Shannon and Simpson diversity measures; e Principle Coordinates Analysis (PCoA) based on Bray-Curtis dissimilarities of salivary microbiome. Axes are scaled to the amount of variation explained; *** $<0.001$

participants who suffer from bleeding gum have significantly higher Bacteroidetes at the phylum level and more Prevotella at the genus level (Fig. 3a, c-upper panel). On the other hand, a significant increase in Actinobacteria, Firmicutes, Proteobacteria and Saccharibacteria phyla was observed in the control participants. Streptococcus, Veillonella, Haemophilus, Granulicatella and Lautrophia genera among others were significantly more abundant in the control group (Fig. 3b, c-lower panel). All indices of alpha diversity indicated that the salivary microbiome in the participants suffering from a bleeding gum is less diverse compared to the controls $\left({ }^{* * *} \mathrm{P}<0.01\right.$, $\left.{ }^{* * * *} \mathrm{P}<0.001\right)$ (Fig. 3d). In the Bray-Curtis based beta diversity, the anosim analysis of distance matrices revealed that there is a significant difference among the two groups with a $P$ value of 0.001 (Fig. 3e). Similar results were also observed in participants that reported having painful gum (a total of 81 participants) or loose teeth (a total of 38 

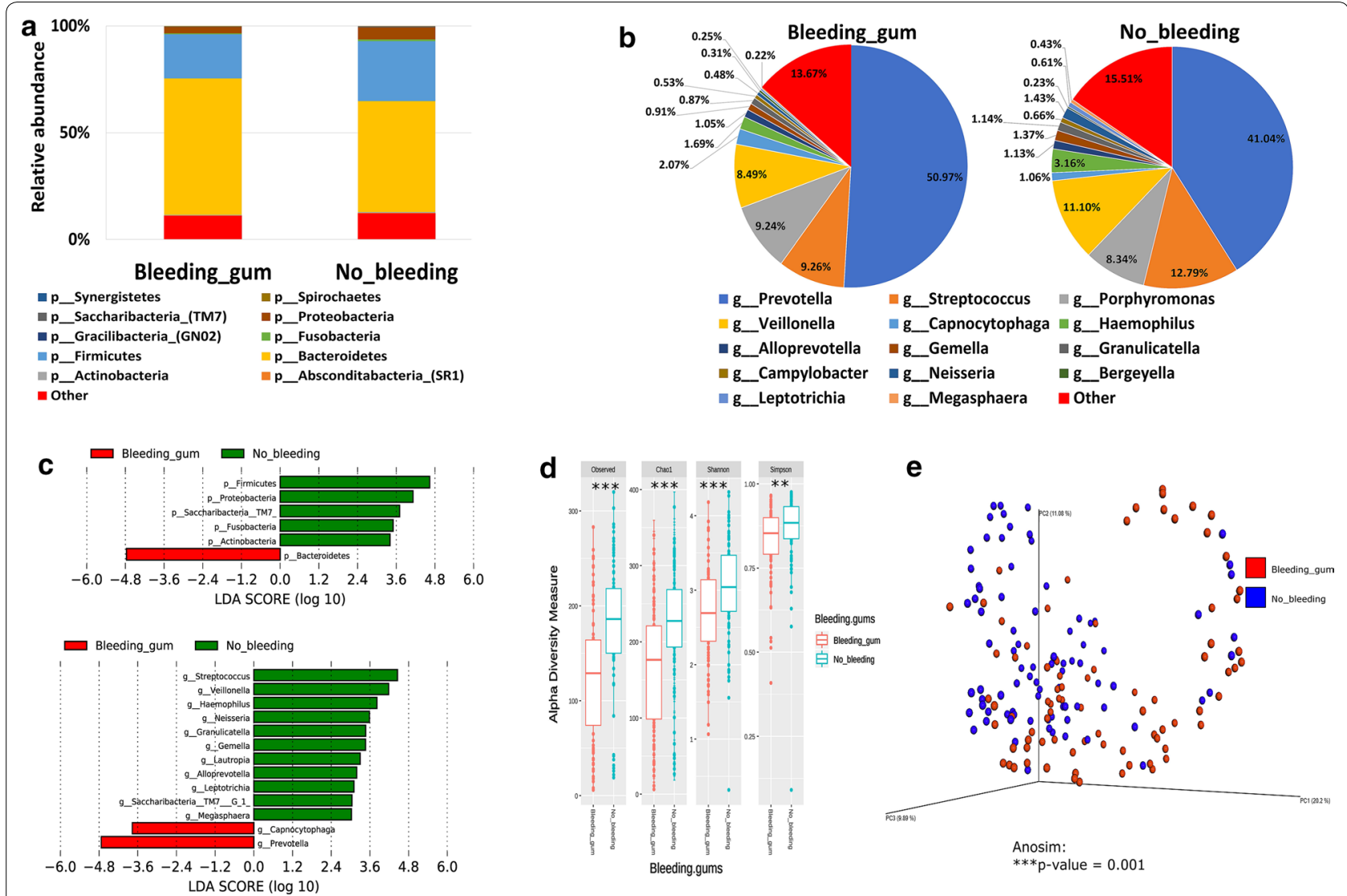

Fig. 3 Bleeding gum and the salivary microbiome composition. Y-axis shows \% of relative abundance; X-axis indicates the microbial abundance in males and females; each taxonomic category is shown by a different color: $\mathbf{a}$ at the phylum level; $\mathbf{b}$ at the genus level; c graphs of linear discriminant analysis (LDA) scores for differentially abundant bacterial phyla and genera; among the two groups. LDA scores indicate overrepresented bacteria in individuals that did not report bleeding (green) and the participants that reported bleeding gums (red). Features with LDA scores $\geq 2$ are presented. $\mathbf{d}$ Alpha diversity measures for the two groups. Alpha diversity was measured by the number of OTUs observed or by the Chao1, Shannon and Simpson diversity measures; e Principle Coordinates Analysis (PCoA) based on Bray-Curtis dissimilarities of salivary microbiome. Axes are scaled to the amount of variation explained; ${ }^{* *} P<0.01$, ${ }^{* *} P<0.001$

participants) (Additional file 3: Figure S3 and Additional file 4: Figure S4).

\section{Mouth ulceration and the salivary microbiome composition}

A total of 37 participants reported suffering from mouth ulcers (Table 1). The salivary microbiome composition in those participants was compared to age and gendermatching controls. Our analysis revealed that Bacteroidetes were significantly higher at the phyla level in participants suffering from mouth ulcers (Fig. 4a, cupper panel), and significantly more Prevotella and Capnocytophaga were observed at the genus level (Fig. 4b, c-lower panel). Similar to the group of participants suffering from bleeding gums, our data show that Actinobacteria, Firmicutes, Proteobacteria and Saccharibacteria at the phyla level and Streptococcus, Veillonella, Haemophilus, Gemella, Granulicatella, Megasphaera and Leptotrichia genera were significantly abundant in the control group (Fig. 4c-lower panel). All indices of alpha diversity indicated that the salivary microbiome in the participants suffering from mouth ulcers is less diverse compared to the controls $\left({ }^{* * *} \mathrm{P}<0.001\right)$ (Fig. $4 \mathrm{~d}$ ). In the Bray-Curtis based beta diversity, the anosim analysis of distance matrices revealed that there is a significant difference among the two groups with a $P$ value of 0.001 (Fig. 4e).

\section{Denture use and the salivary microbiome composition}

A total of 136 participants reported using dentures (Table 1). The salivary microbiome composition in those participants was compared to age and gender-matching controls. Our analysis revealed that Proteobacteria and Actinobacteria were significantly higher at the phylum level in participants using dentures (Fig. 5a, c-upper panel), whereas significantly more Streptococcus, Neisseria and Pseudoramibacter were observed at the genus level (Fig. 5b, 


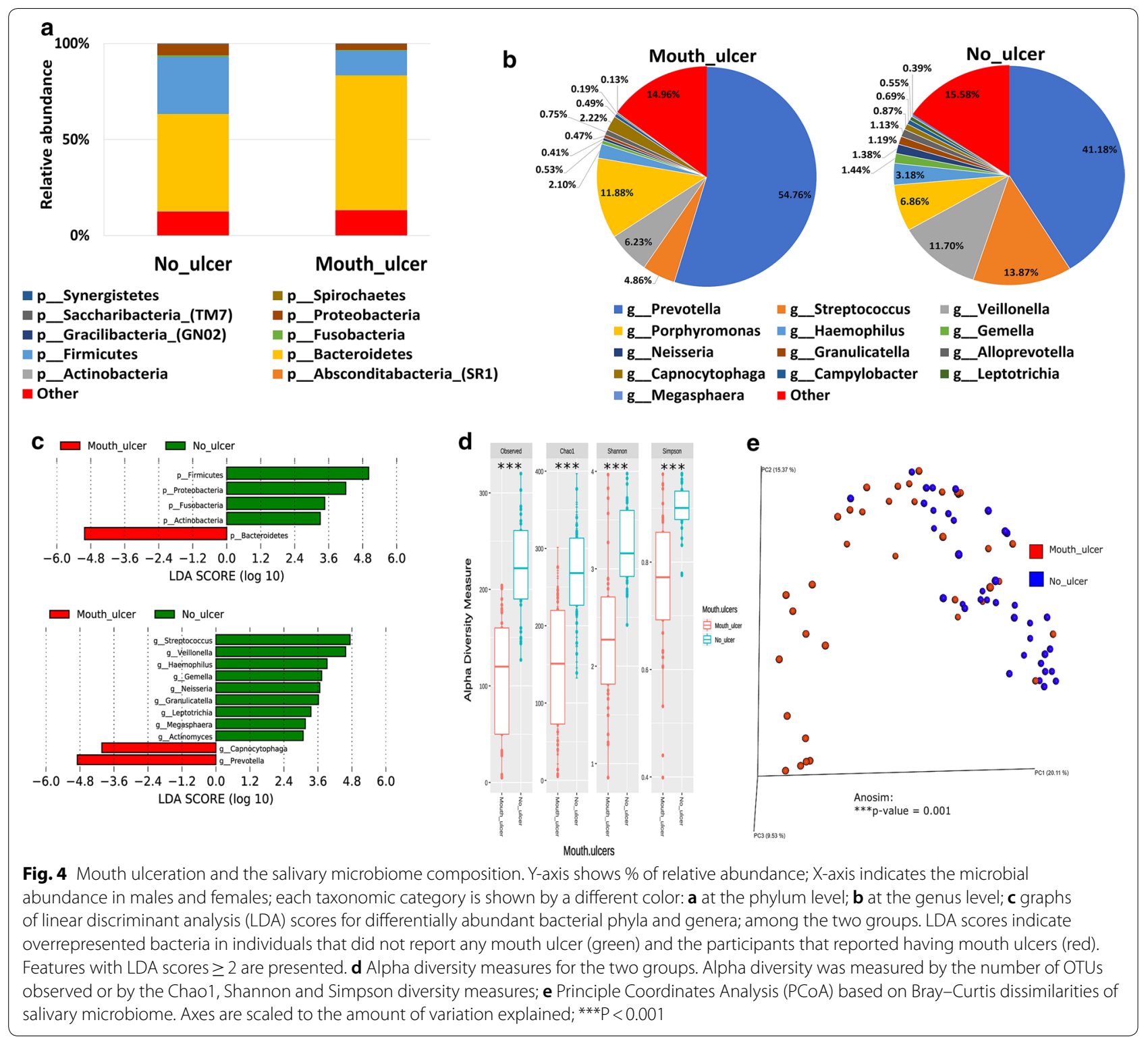

c-lower panel). Moreover, our data show that Campylobacter and Ruminococcaceae genera were significantly higher in the control group (Fig. 5c-lower panel). All indices of alpha diversity indicated that the salivary microbiome in the participants using dentures is less diverse compared to the controls ( ${ }^{*} \mathrm{P}<0.05$, ${ }^{* * *} \mathrm{P}<0.01$, $\left.{ }^{* * * *} \mathrm{P}<0.001\right)$ (Fig. 5d). In the Bray-Curtis based beta diversity, the anosim analysis of distance matrices revealed that there is a significant difference among the two groups with a $P$ value of 0.001 (Fig. 5e).

\section{The salivary microbiome composition is influenced by smoking habits}

Based on their smoking habits, participants were classified into smokers (264 participants) and non-smokers (733 participants). By comparing the two groups, our analysis revealed that Bacteroidetes at the phylum level and Prevotella genus were significantly higher in the smokers' group (Fig. 6a-c). On the other hand, Proteobacteria and Synergistetes at the phylum level, Lactococcus, Corynebacterium, Gemella, Capnocytophaga and 


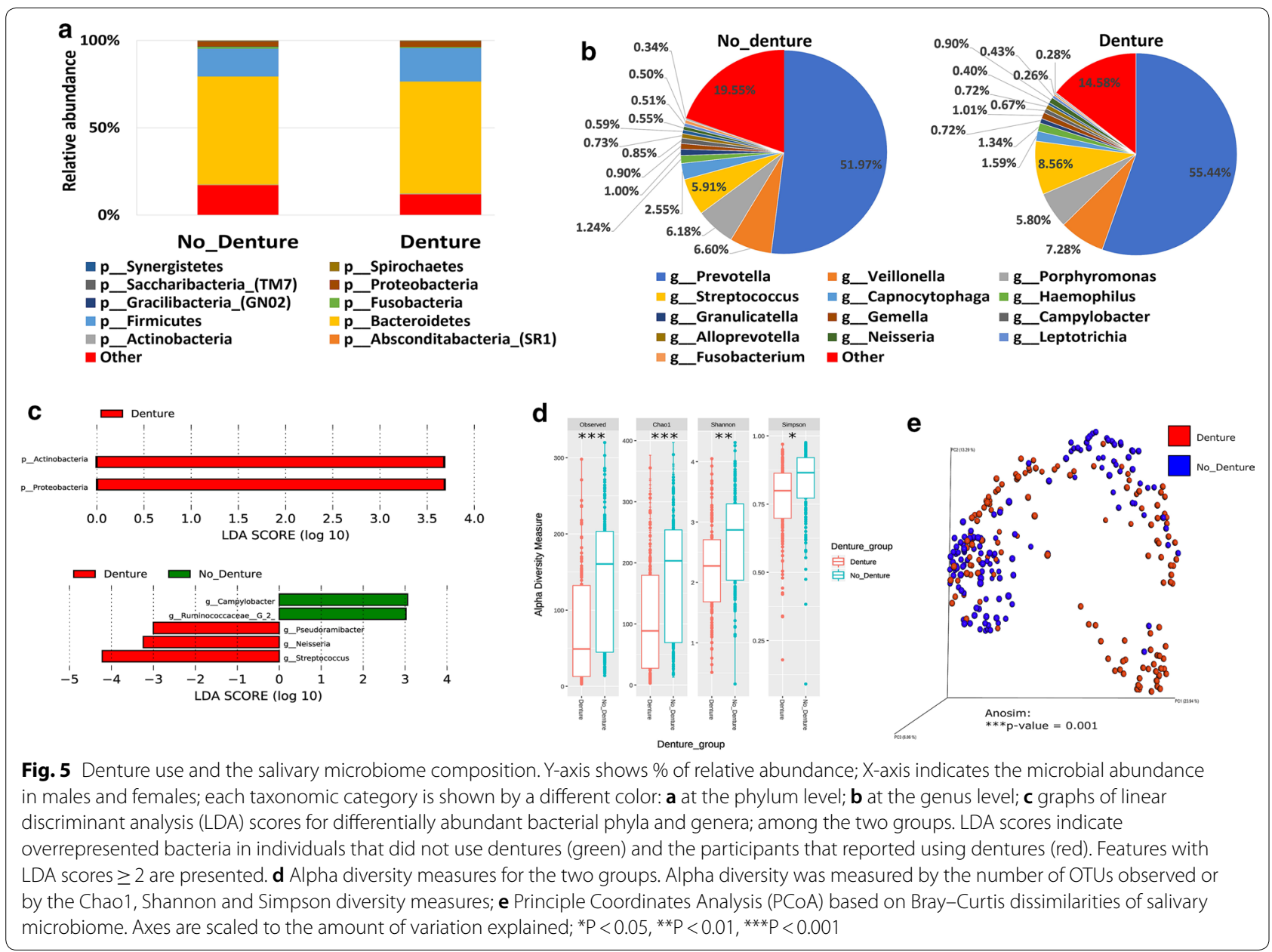

Streptococcus at the genus level were significantly higher in the non-smokers (Fig. 6a-c). The alpha diversity measures indicated that the salivary microbiome of the nonsmokers is significantly more diverse as compared to the smokers ( $\mathrm{P}<0.05)$, but there was no significant difference observed in species richness between the two groups (Fig. 6d). On the other hand, beta diversity measures did not show any significant clustering or distance difference between the two groups (Fig. 6e).

\section{Influence of coffee and tea consumption on the salivary microbiome composition}

The frequency of coffee and tea intake including Arabic coffee, instant coffee, filtered coffee, cappuccino, red tea, herbal tea and Karak (special type of tea mixed with milk, usually offered in the Arabian Gulf countries) were assessed using the dietary questionnaire. From the cohort, the participants were further derived into two groups of coffee/tea drinkers (consuming more than 1-3 cups per week) and non-drinkers (consuming less than 1-3 cups of tea/coffee per month). Among the participants, 110 were coffee drinkers, 887 were noncoffee drinkers, 229 participants were tea drinkers and 768 were non-tea drinkers. The salivary microbiome composition was analyzed in the coffee/tea drinkers and non-drinkers in order to assess the effect of tea and coffee consumption on the salivary microbiome composition.

\section{Coffee consumption}

Our data shows that Actinobacteria, Firmicutes, Proteobacteria and Saccharibacteria phyla were significantly higher in the saliva samples collected from coffee drinkers as compared to the non-coffee drinkers, whereas, Bacteroidetes and Fusobacteria were the significant phyla in the non-coffee drinkers (Fig. 7a, c-upper panel). At the genus level, Streptococcus, Veillonella, Haemophilus, Gemella, Granulicatella and Lautrophia were significantly abundant in the saliva samples collected from coffee drinkers while Prevotella was the most significantly abundant genus observed in the non-coffee drinkers (Fig. 7b, c-lower panel). All indices of alpha diversity indicated that the salivary microbiome in the non-coffee 

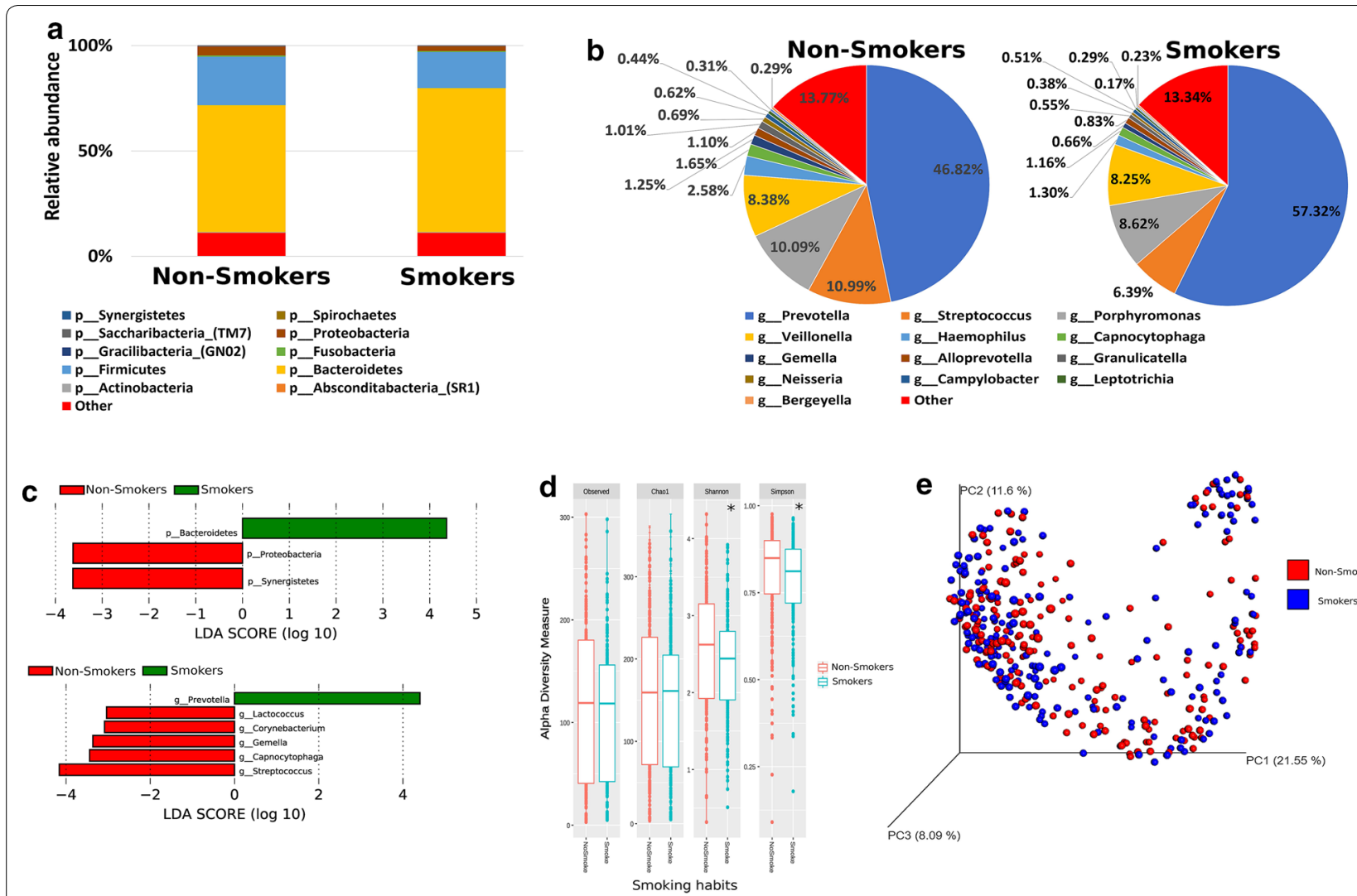

Fig. 6 The salivary microbiome composition is influenced by smoking habits. Y-axis shows \% of relative abundance; X-axis indicates the microbial abundance in males and females; each taxonomic category is shown by a different color: $\mathbf{a}$ at the phylum level; $\mathbf{b}$ at the genus level; c graphs of linear discriminant analysis (LDA) scores for differentially abundant bacterial phyla and genera; among the two groups. LDA scores indicate overrepresented bacteria in smokers (green) and non- smokers (red). Features with LDA scores $\geq 2$ are presented. $\mathbf{d}$ Alpha diversity measures for the two groups. Alpha diversity was measured by the number of OTUs observed or by the Chao1, Shannon and Simpson diversity measures; e Principle Coordinates Analysis (PCOA) based on Bray-Curtis dissimilarities of salivary microbiome. Axes are scaled to the amount of variation explained; ${ }^{*} \mathrm{P}<0.05$

drinkers is less diverse compared to the coffee drinkers $\left({ }^{* * * *} \mathrm{P}<0.001\right)$ (Fig. $7 \mathrm{~d}$ ). In the Bray-Curtis based beta diversity measure, the anosim analysis of distance matrices revealed that there is a significant difference among the two groups with a $P$ value of 0.001 (Fig. 7e).

\section{Tea consumption}

Our data shows that although the abundance of Actinobacteria, Bacteroidetes, Firmicutes and Proteobacteria was higher in the coffee drinkers (Fig. 7a, c-upper panel), no significant difference was observed in the tea drinkers (Fig. 8a, c). At the genus level Capnocytophaga was significantly higher in the non- tea drinkers' group in comparison with the tea drinkers (Fig. 8b, c). The alpha diversity measures showed that a significant increase in the microbial richness was observed in the tea drinking group, ( $\left.{ }^{*} \mathrm{P}<0.05,{ }^{* *} \mathrm{P}<0.01\right)$ but no changes were observed in the microbial diversity indices (Fig. 8d). In the Bray-Curtis based beta diversity measure, the anosim analysis of distance matrices revealed that there is a significant difference among the two groups with a $P$ value of 0.029 (Fig. 8e).

\section{Discussion}

The purpose of this study was to examine the salivary microbiome composition in the Qatari population, and assess its association with gender, age, oral health, smoking coffee and tea consumption. We characterized the salivary microbiome of 997 Qatari participants including 442 males and 555 females. To the best of our knowledge, this is one of the largest population-based studies assessing the salivary microbiome and is the first study to characterize the salivary microbiome in an Arab population like Qataris.

We show that the bacterial profile, in spite of its high diversity, was dominated by the phylum Bacteroidetes, which is different compared to other populations like Bangladesh, UK, Japan, South Korea and Brazil, where 

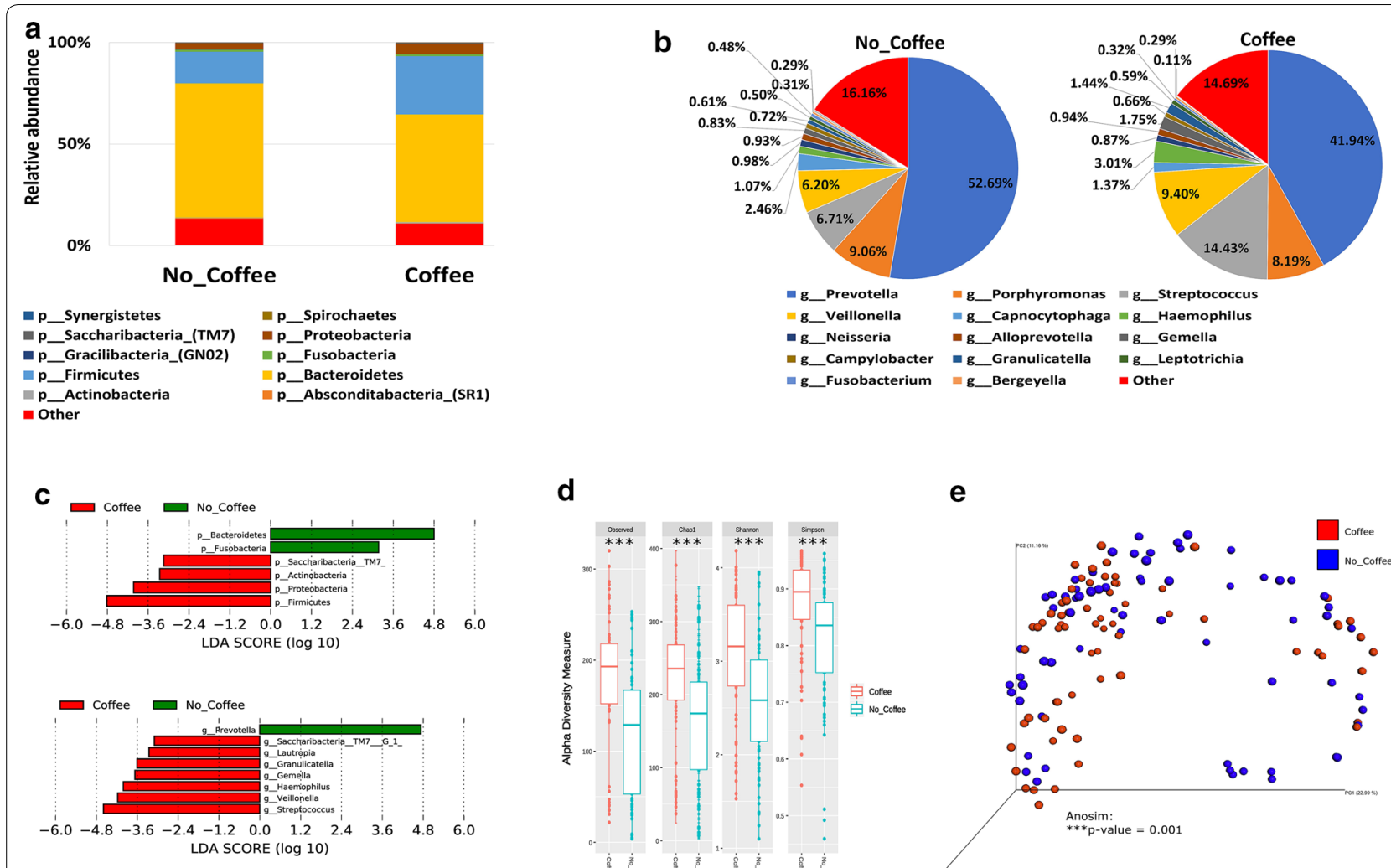

d

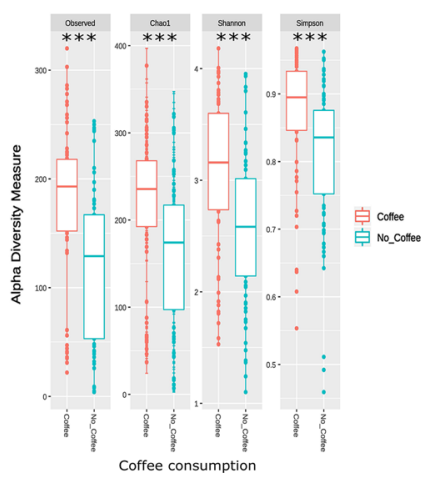

e

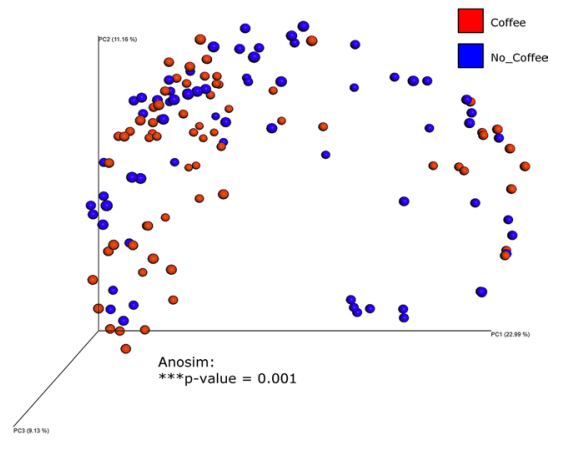

Fig. 7 Influence of Coffee consumption on the salivary microbiome composition. Y-axis shows \% of relative abundance; X-axis indicates the microbial abundance in males and females; each taxonomic category is shown by a different color: $\mathbf{a}$ at the phylum level; $\mathbf{b}$ at the genus level; c graphs of linear discriminant analysis (LDA) scores for differentially abundant bacterial phyla and genera; among the two groups. LDA scores indicate overrepresented bacteria in individuals that do not drink coffee (green) and the participants that are considered coffee drinkers (red). Features with LDA scores $\geq 2$ are presented. $\mathbf{d}$ Alpha diversity measures for the two groups. Alpha diversity was measured by the number of OTUs observed or by the Chao1, Shannon and Simpson diversity measures; e Principle Coordinates Analysis (PCoA) based on Bray-Curtis dissimilarities of salivary microbiome. Axes are scaled to the amount of variation explained; ${ }^{* * *} P<0.001$

Firmicutes was the most predominant phylum. In this study, we show that Prevotella, Porphyromonas, Streptococcus, Veillonella, Haemophilus, Gemella, and Neisseria were the most common members of the Qatari salivary microbiome, with Prevotella being the most predominant genus. In contrast, Streptococcus is the most abundant genus in the UK, South Korea, Japan and US populations [46, 48-53]. The differences observed in the Qatari salivary microbiome compared to other populations, may be influenced by various factors, including host genetics, diet and environmental factors [49].

In this study, we show that the observed species richness index was significantly higher among Qatari males than females, with a significant shift in particular genera like Bergeyella, Tannerella in males and Treponema, Mycoplasma and Corynebacterium in females. This may be influenced by many factors including hormones and body mass index as previously reported [50, 54-56].
Our data show that the salivary microbial composition is associated with age, which is in accordance to previously reported results [57]. The senior participants included in our cohort showed a reduced bacterial diversity and significantly increased Prevotella, when compared to the younger adults. Proteolytic bacteria such as Prevotella are known to degrade proteins and peptides and are associated with periodontitis $[28,58]$. It is known that the saliva composition changes with age due to the slower salivary flow [59], inadequate oral care, increase of several inflammatory mediators [60], systemic diseases and other additional environmental factors that together will affect the microbial composition in the saliva [28, 61]. The adult salivary microbial population this cohort was more diverse including microbes like Streptococcus, Haemophilus, Rothia and Veillonella and Lautrophia, some are known to degrade carbohydrates [50].

We also show that participants that suffer from various oral conditions including bleeding or painful gum, 

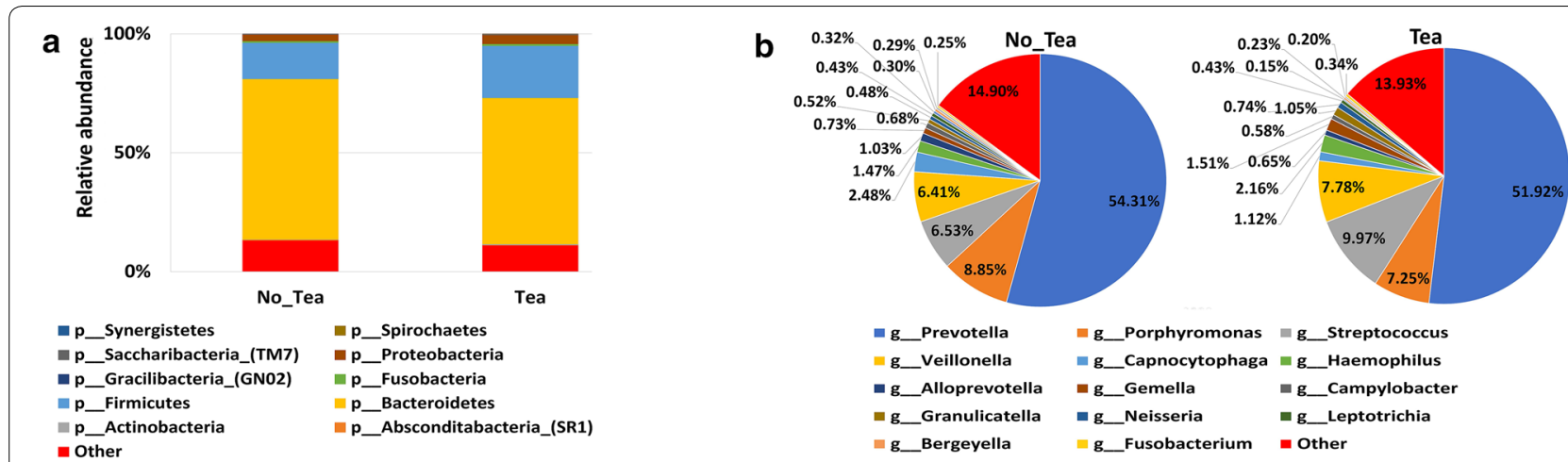

$$
\begin{aligned}
& \text { Q g_Prevotella } \quad \text { g__Porphyromonas }=\mathrm{g} \text { _Streptococcus } \\
& \text { E__Veillonella } \quad \text { g__Capnocytophaga }=\text { g__Haemophilus } \\
& \text { - g_Alloprevotella g_Gemella }=\text { g__Campylobacter } \\
& \text { - g_Granulicatella }=\text { g__Neisseria } \quad \text { g_Leptotrichia } \\
& \text { I__Bergeyella }=\text { g__fusobacterium }=\text { Other }
\end{aligned}
$$
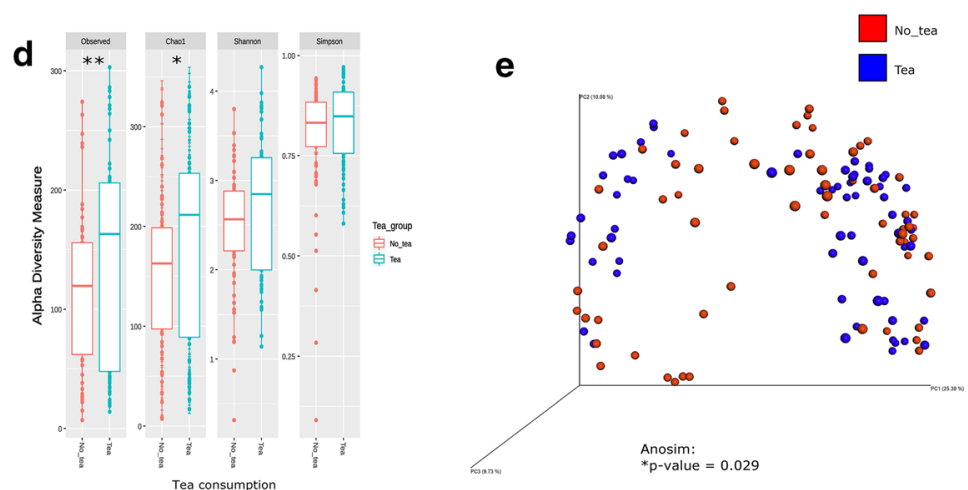

Fig. 8 Influence of tea consumption on the salivary microbiome composition. Y-axis shows \% of relative abundance; X-axis indicates the microbial abundance in males and females; each taxonomic category is shown by a different color: $\mathbf{a}$ at the phylum level; $\mathbf{b}$ at the genus level; $\mathbf{c}$ Graphs of linear discriminant analysis (LDA) scores for differentially abundant bacterial phyla and genera; among the two groups. Features with LDA scores $\geq 2$ are presented. d Alpha diversity measures for the two groups. Alpha diversity was measured by the number of OTUs observed or by the Chao 1, Shannon and Simpson diversity measures; e Principle Coordinates Analysis (PCoA) based on Bray-Curtis dissimilarities of salivary microbiome. Axes are scaled to the amount of variation explained; ${ }^{*} P<0.05,{ }^{*} P<0.01$

loose teeth or mouth ulcers have lower salivary microbial diversity; with Prevotella being the most predominant member. Previous studies have shown an association between increased abundance of Prevotella, aphthous ulcers and periodontal disease (more specifically gingivitis) $[62,63]$. Our data indicates that the alpha diversity of the salivary microbiome in the control groups was significantly higher in comparison to those suffering from poor oral health, which is similar to previously reported results [64]. Our data show that usage of denture is positively correlated with age and is closely linked with the oral health status [65]. In this study, we show that using denture reduces the diversity of the salivary microbiome with an enrichment of both Proteobacteria and Actinobacteria phyla while Streptococcus and Neisseria were enriched at the genus levels. This result is supported by other studies comparing biofilms forming on natural teeth against those forming on denture teeth $[66,67]$.

Smoking modulates the microbial composition of various body sites including upper gut, respiratory tract and the oral microbiome [68-71]. Prior studies had shown that smoking disrupts the microbial homeostasis leading to various oral disease such as gingivitis and dental loss [72]. Our analysis revealed that smoking reduced the salivary microbial diversity and Bacteroidetes was the most abundant phylum observed, which has been reported previously [31]. Moreover, the genus Prevotella was more abundant in the smokers compared to non-smokers, suggesting therefore an increased vulnerability of the smokers to develop oral diseases such as gingivitis [31]. This suggests that smoking has to always be considered in the future when assessing the oral microbiome composition, as it clearly affects the salivary microbiome composition.

Coffee and tea are commonly consumed beverages in most populations, and both were heavily studied to assess their health benefits [73-75]. In our cohort, around 11\% of the Qatari participants reported drinking coffee and $23 \%$ reported drinking tea. While some papers reported that both coffee and tea affect the microbial composition of the saliva [73, 76, 77]; our data show that a significant increase in Granulicatella, Gemella, Streptococcus and Lautrophia along with an increased microbial richness and diversity is observed in the coffee but not in the 
tea drinkers. Higher abundance of Granulicatella in the saliva of coffee drinkers was previously reported [73].

Our data showed that Prevotella is the most abundant bacteria observed in the salivary microbiome of the Qatari adult population. Prevotella is one of the commonly reported members of the oral microbiome [78] and it has been linked previously to various inflammatory conditions such as rheumatoid arthritis, metabolic disorders and periodontal infections among others [79]. Based on gender, we did not observe any significant difference in the abundance of Prevotella between males $(55 \%)$ and females $(53.69 \%)$ in this cohort. We show that, Prevotella was more abundant in subjects with mouth ulcers $(54.76 \%)$, bleeding gum $(50.97 \%)$ as compared to the healthy individuals $(41.04 \%, 41.10 \%)$. Prevotella is higher in the group of smokers (57.32\%) as compared to non-smokers (46.82\%). Various Prevotella species can play different roles in health and disease [79-81]. In a recent study of 161 healthy Italian participants, the salivary microbiome was classified into Prevotelladominant type, Streptococcus/Gemella-dominant type and Neisseria/Fusobacterium-dominant types [82]. The microbial co-occurrence/exclusion pattern was explained by the microorganisms need to nutrients that can be provided by a selective group of bacteria [83, 84]. Our study divulges the decrease in Co-occurring Prevotella/Porphyromonas shifts in healthy controls and increase in diseased cases. In another study Prevotella histicola was shown to have a boosting effect of Copaxone, used to treat patients with multiple sclerosis [85]. Most of the members of Prevotella remain to be considered as commensals in healthy participants, which then turn to pathogens in oral infections and immunocompromised patients. Prevotella is a beneficial microbe that is associated with plant-rich diet and the diverse Prevotella species will have differences in responding to the diet and health status of hosts [86]. More research is needed in order to look further deeper into Prevotella's potential and its interactions with its host and other bacteria for therapeutic use in clinical practice.

\section{Conclusions}

In summary, this large-scale population-based study described for the first time the salivary microbiome profiles in the Qatari population. Our data indicated population-specific microbial composition, with major phyla differences between Qatari population and populations from Brazil, Japan, South Korea, Germany, UK and USA $[12,46,47]$. We also show that the salivary microbiome composition is associated with gender, age, oral health, smoking and coffee intake. The scope of this study is mainly to give a picture of the salivary microbiome and their changes related to various factors. However, due to the limitation of using $16 \mathrm{~S}$ rRNA gene sequencing, we cannot conclude on the function of those microbial changes in relation to various conditions. Future work on the microbial transcriptomics and metabolomics is needed to provide deeper insights into the role of the salivary microbiome in health and disease.

\section{Materials and methods}

\section{Ethics statement}

The study was approved by the Institutional Review board (IRB) of Sidra Medicine under (protocol \#1510001907) and by Qatar Biobank (QBB) under protocol (E/2017/ RES-ACC-0046/0003). All study participants signed an informed consent prior to sample collection. All experiments were performed in accordance with the approved guidelines.

\section{Clinical data}

In this project, an agreement between $\mathrm{QBB}$ and Sidra Medicine was signed in order to collect de-identified salivary samples, phenotypic and clinical data from a total of 997 Qatari participants that were selected randomly. All participants were 18 years old and above. No exclusion criteria were applied in this studied cohort. The cohort consisted of 442 males and 555 females (Table 1). All participants answered the baseline questionnaire to describe their oral hygiene, smoking and dietary habits.

\section{Sample collection}

Saliva samples were collected in QBB according to a standard technique. A total of $5 \mathrm{~mL}$ of spontaneous, whole, unstimulated saliva was collected into a $50 \mathrm{~mL}$ sterile DNA-free Falcon tube from each participant. The samples were divided into $0.4 \mathrm{~mL}$ aliquots and stored at $-80{ }^{\circ} \mathrm{C}$ until further analysis. The aliquots were received from $\mathrm{QBB}$ for total salivary DNA extraction.

\section{DNA extraction and 16S rRNA gene sequencing}

The total salivary DNA were extracted using automated QIAsymphony protocol (Qiagen, Hilden, Germany) following the manufacturer's instructions. DNA purity was evaluated by the A260/A280 ratio using a NanoDrop 7000 Spectrophotometer (Thermo Fisher Scientific, Waltham, MA, USA), and the DNA integrity was checked on a $1 \%$ agarose by gel electrophoresis.

The V1-V3 regions of the 16S rRNA gene were amplified using various forward primers: $27 \mathrm{~F}$ with 12 bp golay barcodes containing a specific Illumina $5^{\prime}$ adapter for each sample and a common reverse primer $515 \mathrm{R}[87,88]$. In brief, PCR was performed in triplicate in a $50 \mu \mathrm{L}$ reaction mixture containing $10 \mathrm{ng}$ of template DNA and $2 \mathrm{x}$ Phusion HotStart Ready Mix (Thermo Scientific ${ }^{\mathrm{TM}}$ ). The following thermal cycling conditions were used: $5 \mathrm{~min}$ of 
initial denaturation at $94{ }^{\circ} \mathrm{C} ; 25$ cycles of denaturation at $94{ }^{\circ} \mathrm{C}$ for $30 \mathrm{~s}$, annealing at $62{ }^{\circ} \mathrm{C}$ for $30 \mathrm{~s}$, extension at $72{ }^{\circ} \mathrm{C}$ for $30 \mathrm{~s}$; and a final extension at $72{ }^{\circ} \mathrm{C}$ for $10 \mathrm{~min}$. The amplified PCR products of approximately $650 \mathrm{bp}$ in size from each sample were pooled in equimolar concentrations according to the manufacturer's instructions (Illumina, Inc., San Diego, CA, USA). This pooled PCR product was purified using AgenCourt AMPure XP magnetic beads (Beckman Coulter). High throughput sequencing was performed on an Illumina MiSeq2 2300 platform in accordance with the Manufacturer's instructions. Image analysis and base calling were carried out directly on the MiSeq.

\section{Taxonomic classification of the salivary microbiome}

Sequenced data were demultiplexed using MiSeq Control Software (MCS). Demultiplexed data were revised for quality control using FastQC [89]. Forward and reverse end sequences of respective samples were merged through PEAR tool [90] and sequence reads of quality score $<20$ were discarded. All merged reads were trimmed to $160 \mathrm{bp}>$ Reads $<500 \mathrm{bp}$ using Trimmomatic tool [91]. Trimmed FASTQ files were converted into FASTA files. Demultiplexed FASTA files were analyzed using QIIME (Quantitative Insights Into Microbial Ecology) v1.9.0 pipeline [92]. Operational taxonomic units (OTUs) were generated by aligning against the Human Oral Microbiome Database (HOMD RefSeq, Version 15.1) with a confidence threshold of $97 \%$ [93]. For the comparison of salivary microbiome profiles, Sequence Read Archive (SRA) files from the bioprojects of other populations such as Brazil (PRJNA504439), Bangladesh (PRJEB23323), United States of America (USA) (PRJNA421776), South Korea (PRJDB2879), Germany (PRJNA387918), United Kingdom (UK) (PRJEB9010) and Japan (PRJDB4107) were retrieved. The Operational taxonomic units (OTUs) of different population were generated by aligning against Greengenes Database (Version:gg_13_8) with a confidence threshold of 97\%.

\section{Significant abundances of the salivary microbiome and diversity analyses}

Linear Discriminant Analysis Effect Size (LEfSe) [94] was used to find differentially abundant taxa between the studied categories, with per-sample normalization to 1 million, an alpha cut-off value of 0.05 for the KruskalWallis factorial test, and a threshold for discriminative features at a logarithmic LDA score $>2$. Alpha diversity was measured by $\mathrm{R}$ software, using the phyloseq package [95]. Beta diversity was represented using Phylogenetic beta diversity metrics [96] and non-phylogenetic beta diversity metrics and the differences in the beta diversity were presented as principle coordinate analysis using QIIME.

\section{Statistical analysis}

Statistical significance of the alpha diversity measures such as Observed, Chao1, Shannon and Simpson indices were calculated using minitab 17 (Minitab statistical software). P-values lower than 0.05 were considered statistically significant. Analysis of similarities software (Anosim) was used to calculate the distance matrix difference between the categories included in this study (adult versus elderly, males versus females, etc.) using Bray-Curtis beta diversity parameters [92].

\section{Supplementary information}

Supplementary information accompanies this paper at https://doi. org/10.1186/s12967-020-02291-2.

Additional file 1: Figure S1. The salivary microbiome of the Qatari population Relative abundance of the total cohort. Y-axis shows \% of relative abundance; $X$-axis indicates the abundance for Qatari population; each taxonomic category is shown by a different color A) phylum level B) genus level.

Additional file 2: Figure S2. The salivary microbiome of the other national population. A) Y-axis shows the percentage of relative abundance; $X$-axis reflects various populations included. Colors in the bar graph reflect bacterial phyla. B) Relative abundance table of the salivary microbiome in various populations at phylum level C) Principle Coordinates Analysis ( $P C O A)$ based on Bray-Curtis dissimilarities of the salivary microbiome. Axes are scaled to the amount of variation explained; ${ }^{* * *} \mathrm{P}<0.001$.

Additional file 3: Figure S3. Salivary microbiome of Participants with Painful gum. Y-axis shows \% of relative abundance; $X$-axis indicates the abundance; each taxonomic category is shown by a different color a) phylum level b) genus level c) Graphs of linear discriminant analysis (LDA) scores for differentially abundant bacterial phyla and genera; among the groups. LDA scores indicate overrepresented bacteria in each group. Features with LDA scores $\geq 2$ are presented. d) Alpha diversity measures were used to compare the two groups. Alpha diversity was measured by the number of OTUs observed, by the Chao1 index, in addition to Shannon and Simpson diversity measures, e) Principle Coordinates Analysis (PCoA) based on Bray-Curtis dissimilarities of the salivary microbiome. Axes are scaled to the amount of variation explained; ${ }^{* * *} \mathrm{P}<0.001$.

Additional file 4: Figure S4. Salivary microbiome of participants with Loose teeth. Y-axis shows \% of relative abundance; $X$-axis indicates the abundance; each taxonomic category is shown by a different color a) phylum level b) genus level c) Graphs of linear discriminant analysis (LDA) scores for differentially abundant bacterial phyla and genera; among the groups. LDA scores indicate overrepresented bacteria in each group. Features with LDA scores $\geq 2$ are presented. d) Alpha diversity measures were used to compare the two groups. Alpha diversity was measured by the number of OTUs observed, by the Chao1 index, Shannon and Simpson diversity measures, e) Principle Coordinates Analysis (PCoA) based on Bray-Curtis dissimilarities of salivary microbiome. Axes are scaled to the amount of variation explained; ${ }^{* *} P<0.01,{ }^{* * *} \mathrm{P}<0.001$.

\section{Acknowledgements}

Data and samples used in this research were obtained from the Qatar Biobank for Research http://www.qatarbiobank.org.qa. Sample processing for DNA extraction was performed by Omics core at Sidra Medicine. Sample processing for DNA sequencing was performed in Sidra Medicine and Qatar Biomedical Research Institute. 


\section{Authors' contributions}

SAK designed the study. SM, SA, MS, PS, MK processed the samples. SM performed the data analysis. SM wrote the first draft. SAK reviewed the data and the final manuscript. All authors read and approved the final manuscript.

\section{Funding}

This project is financially supported by funds from Sidra Medicine to Dr. Souhaila Al Khodor.

\section{Availability of data and materials}

The datasets used and/or analyzed during the current study are available upon request and after approval of QBB.

\section{Ethics approval and consent to participate}

The study was approved by the Institutional Review board (IRB) of Sidra Medicine under (protocol \#1510001907) and by Qatar Biobank (QBB) under protocol (E/2017/RES-ACC-0046/0003). All study participants signed an informed consent prior to sample collection. All experiments were performed in accordance with the approved guidelines.

\section{Consent for publication}

All authors reviewed the final version of the manuscript and approved it for publication

\section{Competing interests}

The authors declare no conflict of interests.

\section{Author details}

${ }^{1}$ Research Department, Sidra Medicine, Doha, Qatar. ${ }^{2}$ College of Health Sciences, Qatar University, Doha, Qatar.

Received: 16 October 2019 Accepted: 4 March 2020

Published online: 14 March 2020

\section{References}

1. Lloyd-Price J, Abu-Ali G, Huttenhower $C$. The healthy human microbiome. Genome Med. 2016;8:51.

2. Liang D, Leung RK, Guan W, Au WW. Involvement of gut microbiome in human health and disease: brief overview, knowledge gaps and research opportunities. Gut Pathog. 2018;10:3.

3. Cao Y, Fanning S, Proos S, Jordan K, Srikumar S. A review on the applications of next generation sequencing technologies as applied to foodrelated microbiome studies. Front Microbiol. 1829;2017:8.

4. Costello EK, Lauber CL, Hamady M, Fierer N, Gordon Jl, Knight R. Bacterial community variation in human body habitats across space and time. Science. 2009;326:1694-7.

5. Roblegg E, Coughran A, Sirjani D. Saliva: an all-rounder of our body. Eur J Pharm Biopharm. 2019;142:133-41.

6. Humphrey SP, Williamson RT. A review of saliva: normal composition, flow, and function. J Prosthet Dent. 2001;85:162-9.

7. Mandel ID. The functions of saliva. J Dent Res. 1987;66 Spec No:623-7.

8. Kaczor-Urbanowicz KE, Martin Carreras-Presas C, Aro K, Tu M, GarciaGodoy F, Wong DT. Saliva diagnostics — current views and directions. Exp Biol Med (Maywood). 2017;242:459-72.

9. Lee YH, Wong DT. Saliva: an emerging biofluid for early detection of diseases. Am J Dent. 2009;22:241-8.

10. Kodukula K, Faller DV, Harpp DN, Kanara I, Pernokas J, Pernokas M, Powers WR, Soukos NS, Steliou K, Moos WH. Gut microbiota and salivary diagnostics: the mouth is salivating to tell us something. BioResearch open access. 2017;6:123-32.

11. Belstrøm D, Holmstrup P, Bardow A, Kokaras A, Fiehn N-E, Paster BJ. Temporal stability of the salivary microbiota in oral health. PLOS ONE. 2016;11:e0147472.

12. Cameron SJ, Huws SA, Hegarty MJ, Smith DP, Mur LA. The human salivary microbiome exhibits temporal stability in bacterial diversity. FEMS Microbiol Ecol. 2015;91:fiv091.

13. Rasiah IA, Wong $L$, Anderson $S A$, Sissons $C H$. Variation in bacterial DGGE patterns from human saliva: over time, between individuals and in corresponding dental plaque microcosms. Arch Oral Biol. 2005;50:779-87.
14. Stahringer SS, Clemente JC, Corley RP, Hewitt J, Knights D, Walters WA, Knight R, Krauter KS. Nurture trumps nature in a longitudinal survey of salivary bacterial communities in twins from early adolescence to early adulthood. Genome Res. 2012;22:2146-52.

15. Yamanaka W, Takeshita T, Shibata Y, Matsuo K, Eshima N, Yokoyama T, Yamashita Y. Compositional stability of a salivary bacterial population against supragingival microbiota shift following periodontal therapy. PLOS ONE. 2012;7:e42806.

16. Zhou Y, Gao H, Mihindukulasuriya KA, La Rosa PS, Wylie KM, Vishnivetskaya T, Podar M, Warner B, Tarr PI, Nelson DE, et al. Biogeography of the ecosystems of the healthy human body. Genome Biol. 2013;14:R1.

17. Priya KY, Prathibha KM. Methods of collection of saliva-a review. Int J Oral Health Dent. 2017:3:149-53.

18. Lim Y, Totsika M, Morrison M, Punyadeera C. The saliva microbiome profiles are minimally affected by collection method or DNA extraction protocols. Sci Rep. 2017;7:8523.

19. Rosier BT, Marsh PD, Mira A. Resilience of the oral microbiota in health: mechanisms that prevent dysbiosis. J Dent Res. 2018;97:371-80.

20. He J, LiY, Cao Y, Xue J, Zhou X. The oral microbiome diversity and its relation to human diseases. Folia Microbiol (Praha). 2015;60:69-80.

21. Aas JA, Paster BJ, Stokes LN, Olsen I, Dewhirst FE. Defining the normal bacterial flora of the oral cavity. J Clin Microbiol. 2005;43:5721-32.

22. Zaura E, Keijser BJ, Huse SM, Crielaard W. Defining the healthy "core microbiome" of oral microbial communities. BMC Microbiol. 2009:9:259.

23. Bik EM, Long CD, Armitage GC, Loomer P, Emerson J, Mongodin EF, Nelson KE, Gill SR, Fraser-Liggett CM, Relman DA. Bacterial diversity in the oral cavity of 10 healthy individuals. ISME J. 2010;4:962-74.

24. Segata N, Haake SK, Mannon P, Lemon KP, Waldron L, Gevers D, Huttenhower C, Izard J. Composition of the adult digestive tract bacterial microbiome based on seven mouth surfaces, tonsils, throat and stool samples. Genome Biol. 2012;13:R42.

25. Hall MW, Singh N, Ng KF, Lam DK, Goldberg MB, Tenenbaum HC, Neufeld JD. R GB, Senadheera DB: Inter-personal diversity and temporal dynamics of dental, tongue, and salivary microbiota in the healthy oral cavity. NPJ Biofilms Microbiomes. 2017:3:2.

26. Wu Y, Chi X, Zhang Q, Chen F, Deng X. Characterization of the salivary microbiome in people with obesity. PeerJ. 2018;6:e4458.

27. Rusthen S, Kristoffersen AK, Young A, Galtung HK, Petrovski BE, Palm O, Enersen $M$, Jensen JL. Dysbiotic salivary microbiota in dry mouth and primary Sjogren's syndrome patients. PLoS ONE. 2019;14:e0218319.

28. Lira-Junior R, Akerman S, Klinge B, Bostrom EA, Gustafsson A. Salivary microbial profiles in relation to age, periodontal, and systemic diseases. PLoS ONE. 2018;13:e0189374.

29. Krishnan $\mathrm{K}$, Chen T, Paster BJ. A practical guide to the oral microbiome and its relation to health and disease. Oral Dis. 2017;23:276-86.

30. Wu J, Peters BA, Dominianni C, Zhang Y, Pei Z, Yang L, Ma Y, Purdue MP, Jacobs EJ, Gapstur SM, et al. Cigarette smoking and the oral microbiome in a large study of American adults. ISME J. 2016;10:2435-46.

31. Rodríguez-Rabassa M, López P, Rodríguez-Santiago RE, Cases A, Felici M, Sánchez R, Yamamura Y, Rivera-Amill V. Cigarette smoking modulation of saliva microbial composition and cytokine levels. Int J Environ Res Public Health. 2018;15:2479.

32. Li X, Kolltveit KM, Tronstad L, Olsen I. Systemic diseases caused by oral infection. Clin Microbiol Rev. 2000;13:547-58.

33. Yasunaga $H$, Takeshita $T$, Shibata Y, Furuta M, Shimazaki Y, Akifusa S, Ninomiya T, Kiyohara Y, Takahashi I, Yamashita Y. Exploration of bacterial species associated with the salivary microbiome of individuals with a low susceptibility to dental caries. Clin Oral Investig. 2017;21:2399-406.

34. Belstrom D, Holmstrup P, Fiehn NE, Kirkby N, Kokaras A, Paster BJ, Bardow A. Salivary microbiota in individuals with different levels of caries experience. J Oral Microbiol. 2017;9:1270614.

35. Schoilew K, Ueffing H, Dalpke A, Wolff B, Frese C, Wolff D, Boutin S. Bacterial biofilm composition in healthy subjects with and without caries experience. J Oral Microbiol. 2019;11:1633194.

36. Ennibi OK, Claesson R, Akkaoui S, Reddahi S, Kwamin F, Haubek D, Johansson A. High salivary levels of JP2 genotype of Aggregatibacter actinomycetemcomitans is associated with clinical attachment loss in Moroccan adolescents. Clin Exp Dent Res. 2019;5:44-51.

37. Salminen A, Kopra KA, Hyvarinen K, Paju S, Mantyla P, Buhlin K, Nieminen MS, Sinisalo J, Pussinen PJ. Quantitative PCR analysis of salivary pathogen burden in periodontitis. Front Cell Infect Microbiol. 2015:5:69. 
38. Khemwong T, Kobayashi H, Ikeda Y, Matsuura T, Sudo T, Kano C, Mikami $\mathrm{R}$, Izumi Y. Fretibacterium sp. human oral taxon 360 is a novel biomarker for periodontitis screening in the Japanese population. PLoS ONE. 2019;14:e0218266.

39. Janem WF, Scannapieco FA, Sabharwal A, Tsompana M, Berman HA, Haase EM, Miecznikowski JC, Mastrandrea LD. Salivary inflammatory markers and microbiome in normoglycemic lean and obese children compared to obese children with type 2 diabetes. PLOS ONE. 2017;12:e0172647.

40. Acharya A, Chan Y, Kheur S, Jin LJ, Watt RM, Mattheos N. Salivary microbiome in non-oral disease: a summary of evidence and commentary. Arch Oral Biol. 2017;83:169-73.

41. Pereira PAB, Aho VTE, Paulin L, Pekkonen E, Auvinen P, Scheperjans F. Oral and nasal microbiota in Parkinson's disease. Parkinsonism Related Disord. 2017;38:61-7.

42. Karpiński TM. Role of oral microbiota in cancer development. Microorganisms. 2019;7:20.

43. Zhang $X$, Zhang D, Jia H, Feng $Q$, Wang D, Liang D, Wu X, Li J, Tang L, Li Y, et al. The oral and gut microbiomes are perturbed in rheumatoid arthritis and partly normalized after treatment. Nat Med. 2015;21:895.

44. Gao L, Xu T, Huang G, Jiang S, Gu Y, Chen F. Oral microbiomes: more and more importance in oral cavity and whole body. Protein Cell. 2018;9:488-500.

45. Sampaio-Maia B, Caldas IM, Pereira ML, Perez-Mongiovi D, Araujo R. The oral microbiome in health and its implication in oral and systemic diseases. Adv Appl Microbiol. 2016;97:171-210.

46. Takeshita T, Kageyama S, Furuta M, Tsuboi H, Takeuchi K, Shibata Y, Shimazaki Y, Akifusa S, Ninomiya T, Kiyohara Y, Yamashita Y. Bacterial diversity in saliva and oral health-related conditions: the Hisayama Study. Sci Rep. 2016;6:22164.

47. Vogtmann E, Chen J, Kibriya MG, Amir A, Shi J, Chen Y, Islam T, Eunes M, Ahmed A, Naher J, et al. Comparison of oral collection methods for studies of microbiota. Cancer Epidemiol Biomark Prev. 2019;28:137-43.

48. Xun Z, Zhang Q, Xu T, Chen N, Chen F. Dysbiosis and ecotypes of the salivary microbiome associated with inflammatory bowel diseases and the assistance in diagnosis of diseases using oral bacterial profiles. Front Microbiol. 2018;9:1136.

49. Shaw L, Ribeiro ALR, Levine AP, Pontikos N, Balloux F, Segal AW, Roberts AP, Smith AM. The human salivary microbiome is shaped by shared environment rather than genetics: evidence from a large family of closely related individuals. MBio. 2017;8:e01237-17.

50. Raju SC, Lagstrom S, Ellonen P, de Vos WM, Eriksson JG, Weiderpass E, Rounge TB. Gender-specific associations between saliva microbiota and body size. Front Microbiol. 2019;10:767.

51. Cabral DJ, Wurster JI, Flokas ME, Alevizakos M, Zabat M, Korry BJ, Rowan AD, Sano WH, Andreatos N, Ducharme RB, et al. The salivary microbiome is consistent between subjects and resistant to impacts of short-term hospitalization. Sci Rep. 2017;7:11040.

52. Xu X, He J, Xue J, Wang Y, Li K, Zhang K, Guo Q, Liu X, Zhou Y, Cheng L, et al. Oral cavity contains distinct niches with dynamic microbial communities. Environ Microbiol. 2015:17:699-710.

53. Percival RS, Challacombe SJ, Marsh PD. Age-related microbiological changes in the salivary and plaque microflora of healthy adults. J Med Microbiol. 1991;35:5-11.

54. Fransen F, van Beek AA, Borghuis T, Meijer B, Hugenholtz F, van der Gaastde Jongh C, Savelkoul HF, de Jonge MI, Faas MM, Boekschoten MV, et al. The impact of gut microbiota on gender-specific differences in immunity. Front Immunol. 2017:8:754

55. Haro C, Rangel-Zuniga OA, Alcala-Diaz JF, Gomez-Delgado F, Perez-Martinez P, Delgado-Lista J, Quintana-Navarro GM, Landa BB, Navas-Cortes JA, Tena-Sempere M, et al. Intestinal microbiota is influenced by gender and body mass index. PLoS ONE. 2016;11:e0154090.

56. Zaura E, Brandt BW, Mattos MJ, Buijs MJ, Caspers MP, Rashid MU, Weintraub A, Nord CE, Savell A, Hu Y, et al. Same exposure but two radically different responses to antibiotics: resilience of the salivary microbiome versus long-term microbial shifts in feces. MBio. 2015;6:e01693-01615.

57. Takahashi N. Oral microbiome metabolism: from "Who Are They?" to "What Are They Doing?". J Dent Res. 2015;94:1628-37.

58. Frandsen EV, Kjeldsen M, Kilian M. Inhibition of Prevotella and Capnocytophaga immunoglobulin A1 proteases by human serum. Clin Diagn Lab Immunol. 1997:4:458-64.
59. Affoo RH, Foley N, Garrick R, Siqueira WL, Martin RE. Meta-analysis of salivary flow rates in young and older adults. J Am Geriatr Soc. 2015:63:2142-51.

60. Cruz-Almeida Y, Aguirre M, Sorenson H, Tighe P, Wallet SM, Riley JL 3rd. Age differences in salivary markers of inflammation in response to experimental pain: does venipuncture matter? J Pain Res. 2017;10:2365-72.

61. Jiang Q, Liu J, Chen L, Gan N, Yang D. The oral microbiome in the elderly with dental caries and health. Front Cell Infect Microbiol. 2018;8:442.

62. Marchini L, Campos MS, Silva AM, Paulino LC, Nobrega FG. Bacterial diversity in aphthous ulcers. Oral Microbiol Immunol. 2007;22:225-31.

63. Lourenvarsigmao TGB, Spencer SJ, Alm EJ, Colombo APV. Defining the gut microbiota in individuals with periodontal diseases: an exploratory study. J Oral Microbiol. 2018;10:1487741.

64. Mashima I, Theodorea CF, Thaweboon B, Thaweboon S, Scannapieco FA, Nakazawa F. Exploring the salivary microbiome of children stratified by the oral hygiene index. PLOS ONE. 2017;12:e0185274.

65. Shi B, Wu T, McLean J, Edlund A, Young Y, He X, Lv H, Zhou X, Shi W, Li H, Lux R. The denture-associated oral microbiome in health and stomatitis. mSphere. 2016. https://doi.org/10.1128/mSphere.00215-16.

66. O'Donnell LE, Robertson D, Nile CJ, Cross LJ, Riggio M, Sherriff A, Bradshaw D, Lambert M, Malcolm J, Buijs MJ, et al. The oral microbiome of denture wearers is influenced by levels of natural dentition. PLOS ONE. 2015;10:e0137717.

67. Teles FR, Teles RP, Sachdeo A, Uzel NG, Song XQ, Torresyap G, Singh M, Papas A, Haffajee AD, Socransky SS. Comparison of microbial changes in early redeveloping biofilms on natural teeth and dentures. J Periodontol. 2012;83:1139-48

68. Brook I. The impact of smoking on oral and nasopharyngeal bacterial flora. J Dent Res. 2011;90:704-10.

69. Charlson ES, Chen J, Custers-Allen R, Bittinger K, Li H, Sinha R, Hwang J, Bushman FD, Collman RG. Disordered microbial communities in the upper respiratory tract of cigarette smokers. PLoS ONE. 2010;5:e15216.

70. Berkowitz L, Schultz BM, Salazar GA, Pardo-Roa C, Sebastian VP, AlvarezLobos MM, Bueno SM. Impact of cigarette smoking on the gastrointestinal tract inflammation: opposing effects in Crohn's disease and ulcerative colitis. Front Immunol. 2018;9:74.

71. Yu G, Phillips S, Gail MH, Goedert JJ, Humphrys MS, Ravel J, Ren Y, Caporaso NE. The effect of cigarette smoking on the oral and nasal microbiota. Microbiome. 2017;5:3

72. Grover N, Sharma J, Sengupta S, Singh S, Singh N, Kaur H. Long-term effect of tobacco on unstimulated salivary $\mathrm{pH}$. J Oral Maxillofac Pathol. 2016:20:16-9.

73. Peters BA, McCullough ML, Purdue MP, Freedman ND, Um CY, Gapstur SM, Hayes RB, Ahn J. Association of coffee and tea intake with the oral microbiome: results from a large cross-sectional study. Cancer Epidemiol Biomark Prev. 2018:27:814-21.

74. Oz HS. Chronic inflammatory diseases and green tea polyphenols. Nutrients. 2017;9:561.

75. Loftfield E, Shiels MS, Graubard BI, Katki HA, Chaturvedi AK, Trabert B, Pinto LA, Kemp TJ, Shebl FM, Mayne ST, et al. Associations of coffee drinking with systemic immune and inflammatory markers. Cancer Epidemiol Biomark Prev. 2015;24:1052-60.

76. Signoretto C, Bianchi F, Burlacchini G, Sivieri F, Spratt D, Canepari P. Drinking habits are associated with changes in the dental plaque microbial community. J Clin Microbiol. 2010;48:347-56.

77. Signoretto C, Burlacchini G, Bianchi F, Cavalleri G, Canepari P. Differences in microbiological composition of saliva and dental plaque in subjects with different drinking habits. N Microbiol. 2006;29:293-302.

78. Palmer RJ Jr. Composition and development of oral bacterial communities. Periodontol. 2000;2014(64):20-39.

79. Larsen JM. The immune response to Prevotella bacteria in chronic inflammatory disease. Immunology. 2017;151:363-74.

80. Su T, Liu R, Lee A, Long Y, Du L, Lai S, Chen X, Wang L, Si J, Owyang C, Chen $S$. Altered intestinal microbiota with increased abundance of prevotella is associated with high risk of diarrhea-predominant irritable bowel syndrome. Gastroenterol Res Pract. 2018;2018:6961783.

81. Leite AZ, Rodrigues NC, Gonzaga MI, Paiolo JCC, de Souza CA, Stefanutto NAV, Omori WP, Pinheiro DG, Brisotti JL, Matheucci Junior E, et al. Detection of increased plasma interleukin-6 levels and prevalence of Prevotella copri and Bacteroides vulgatus in the feces of type 2 diabetes patients. Front Immunol. 2017:8:1107. 
82. De Filippis F, Vannini L, La Storia A, Laghi L, Piombino P, Stellato G, Serrazanetti DI, Gozzi G, Turroni S, Ferrocino I, et al. The same microbiota and a potentially discriminant metabolome in the saliva of omnivore, ovo-lacto-vegetarian and Vegan individuals. PLOS ONE. 2014;9:e112373.

83. Faust K, Sathirapongsasuti JF, Izard J, Segata N, Gevers D, Raes J, Huttenhower C. Microbial co-occurrence relationships in the human microbiome. PLoS Comput Biol. 2012:8:e1002606.

84. Takeshita T, Nakano Y, Kumagai T, Yasui M, Kamio N, Shibata Y, Shiota S, Yamashita Y. The ecological proportion of indigenous bacterial populations in saliva is correlated with oral health status. ISME J. 2009;3:65-78.

85. Shahi SK, Freedman SN, Murra AC, Zarei K, Sompallae R, Gibson-Corley KN, Karandikar NJ, Murray JA, Mangalam AK. Prevotella histicola, a human gut commensal, is as potent as COPAXONE(R) in an animal model of multiple sclerosis. Front Immunol. 2019;10:462.

86. Kovatcheva-Datchary P, Nilsson A, Akrami R, Lee YS, De Vadder F, Arora T, Hallen A, Martens E, Bjorck I, Backhed F. Dietary fiber-induced improvement in glucose metabolism is associated with increased abundance of prevotella. Cell Metab. 2015;22:971-82

87. Zheng W, Tsompana M, Ruscitto A, Sharma A, Genco R, Sun Y, Buck MJ. An accurate and efficient experimental approach for characterization of the complex oral microbiota. Microbiome. 2015;3:48.

88. Mattei V, Murugesan S, Al Hashmi M, Mathew R, James N, Singh P, Kumar M, Lakshmanan AP, Terranegra A, Al Khodor S, Tomei S. Evaluation of methods for the extraction of microbial DNA from vaginal swabs used for microbiome studies. Front Cell Infect Microbiol. 2019;9:197.

89. Andrews S. FastQC: a quality control tool for high throughput sequence data; 2010.
90. Zhang J, Kobert K, Flouri T, Stamatakis A. PEAR: a fast and accurate Illumina Paired-End reAd mergeR. Bioinformatics. 2014;30:614-20.

91. Bolger AM, Lohse M, Usadel B. Trimmomatic: a flexible trimmer for Illumina sequence data. Bioinformatics. 2014;30:2114-20.

92. Caporaso JG, Kuczynski J, Stombaugh J, Bittinger K, Bushman FD, Costello EK, Fierer N, Peña AG, Goodrich JK, Gordon Jl, et al. QIIME allows analysis of high-throughput community sequencing data. Nat Methods. 2010;7:335-6.

93. Escapa IF, Chen T, Huang Y, Gajare P, Dewhirst FE, Lemon KP. New insights into human nostril microbiome from the expanded human oral microbiome database (eHOMD): a resource for the microbiome of the human aerodigestive tract. Systems. 2018;3:e00187-00118.

94. Segata N, Izard J, Waldron L, Gevers D, Miropolsky L, Garrett WS, Huttenhower C. Metagenomic biomarker discovery and explanation. Genome Biol. 2011;12:R60.

95. McMurdie PJ, Holmes S. phyloseq: an R package for reproducible interactive analysis and graphics of microbiome census data. PLOS ONE. 2013;8:e61217.

96. Lozupone CA, Hamady M, Kelley ST, Knight R. Quantitative and qualitative beta diversity measures lead to different insights into factors that structure microbial communities. Appl Environ Microbiol. 2007;73:1576-85.

\section{Publisher's Note}

Springer Nature remains neutral with regard to jurisdictional claims in published maps and institutional affiliations.
Ready to submit your research? Choose BMC and benefit from:

- fast, convenient online submission

- thorough peer review by experienced researchers in your field

- rapid publication on acceptance

- support for research data, including large and complex data types

- gold Open Access which fosters wider collaboration and increased citations

- maximum visibility for your research: over $100 \mathrm{M}$ website views per year

At BMC, research is always in progress.

Learn more biomedcentral.com/submissions 